sensitive," adds Adams. "It blew me away when I saw the results. We had to repeat it a few times to believe it, but it's real."

Meanwhile, other companies are advancing biomarker detection platforms that similarly rely on mechanical forces, rather than traditional optics, but with technologies that use other types of physics (see "Biomarking the way').

\section{Diagnostic ambitions}

Although Lundstrom is currently focused on tailoring his sensors to detect proteins as part of drug discovery experiments, he hopes to eventually move into the diagnostics arena. In fact, BioScale already has a handheld device in the works for clinical applications. "We would redesign components of the system to be more diagnostics friendly," he explains, as he demos the prototype, which is about the size of Walkman cassette player. "The technology stays the same, but the interface will be completely different."

To get there, however, the company would need to conduct clinical trials and then file for regulatory approval. Although Lundstrom says he's had preliminary discussions with lawyers about going down that route, for now his focus remains drug discovery.

Whether pharmaceutical and academic laboratories are willing to pay for the $\mathrm{ViBE}$ platform remains to be seen. BioScale's bioanalyzer costs $\$ 65,000$ when sold as a standalone component and $\$ 130,000$ when marketed as part of a fully automated workstation. The cost of comparable machines that are typically used in drug discovery is all over the map: for example, MAGPIX, a multiplexed version of ELISA sold by Austin, Texas-based Luminex, is priced at
$\$ 35,000$, whereas Singulex’s Erenna, a singlemolecule-counting variant on a traditional immunoassay, approaches $\$ 300,000$.

Lundstrom claims he is seeing a growing interest in the ViBE machines, and he expects the company to be profitable within a year. "You can probably name most top pharma companies, and they have one in evaluation at the moment," he says.

"It's kind of cool," he adds, "to have a technology that's not only really sensitive but works really well in complex samples." It might take some time for the technology to catch on, but Lundstrom and others developing nonoptical techniques to measure biomarkers hope that drug developers will see the light.

Elie Dolgin is a news editor with Nature Medicine in New York.

\section{Biomarking the way}

Less than a mile away from BioScale, another Lexington, Massachusetts-based company called T2 Biosystems is also developing a biomarker detector that similarly sidesteps the traditional use of light scan samples. Its platform, dubbed the T2Dx, uses both magnetic resonance and nanoparticles to rapidly test for pathogens in whole blood. "There's a big push to move to nonoptical detection," says Tom Lowery, vice president of diagnostics research and discovery with the company. "The question is, "who has the data?'"

T2 thinks they do. At a recent meeting this spring, the company presented data showing that their technology detected fungal pathogens in spiked blood samples with high precision at levels as low as ten fungal cells per milliliter. And, a month later, at the general meeting of the American Society for Microbiology in New Orleans, scientists from T2 reported perfect concordance between the data obtained from the T2Dx platform and the results seen by classic microbiology culturing techniques in a small cohort of

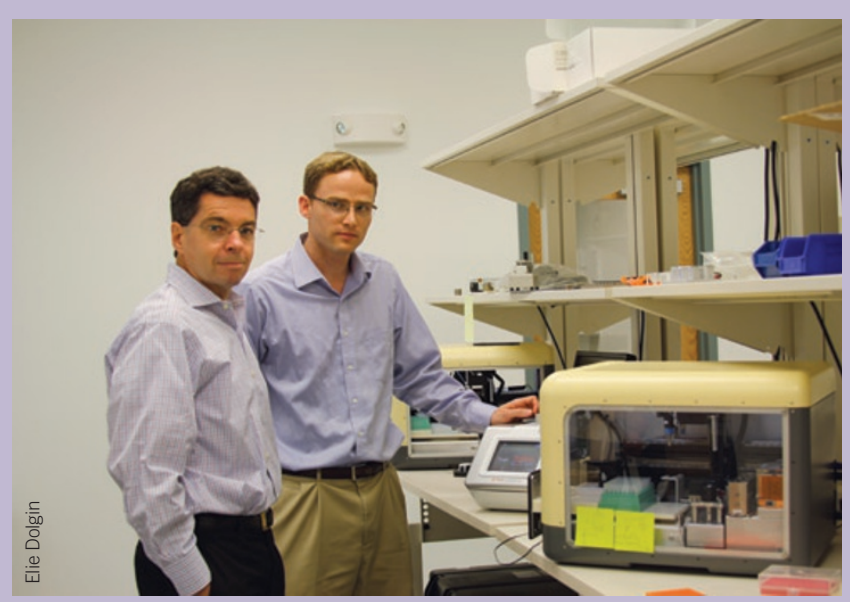

Tea for two: T2's John McDonough and Tom Lowery. people with Candida albicans infections.

"I haven't seen anything more powerful," says T2's chief executive John McDonough. "Companies like Roche have been trying to do direct detection in whole blood for 30 years. We feel that we're in a pretty good position with this technology."

Meanwhile, Nanosphere, a company headquartered in the outskirts of Chicago, also has a nanoparticle-based detection system for protein diagnostics. Theirs is unique in that it relies on minute spheres of gold that, using the company's device, help to quantify the levels of any biomarker of interest.

Currently, the company is developing their technology for two diagnostic purposes: predicting relapse rates of prostate cancer and measuring risk for cardiovascular disease.

In 2009, cofounder Chad Mirkin, a chemist at Northwestern University in Evanston, Illinois, showed that the assay could detect vanishingly low levels of PSA in samples from men taken after radical prostatectomies that commercial immunoassays missed. What's more, the device picked up tiny changes in these PSA levels that allowed the researchers to accurately forecast for whom prostate cancer would recur (Proc. Natl. Acad. Sci. USA 106, 18437-18442, 2009).

Earlier this year, researchers from the University of CaliforniaSan Diego also used Nanosphere's platform to show that troponin I, a key regulator of muscle contraction, was a good predictor of mortality and hospitalization rates in people with severe heart failure (Eur. J. Heart Fail. 13, 37-42, 2011). "Those are a number of brand new applications for biomarkers that are enabled by the level of sensitivity that we achieve in our proprietary nanoparticlebased assay," says Nanosphere chief executive Bill Moffitt.

Onlookers such as Matt Cooper from Australia's University of Queensland see a sea change underway as companies and academic researchers shift away from the traditional technologies toward new types of physics. "As we go to more direct simple measurement of [biomarkers]," he says, "I think you'll see nonoptical approaches be more and more adopted." - $-E D$ 